\title{
Mutations in KCND3 linked to spinocerebellar ataxias
}

The efforts of five independent research groups have identified mutations in the KCND3 gene that cause spinocerebellar ataxia (SCA) type 19 and type 22 . The groups used a combination of genetic linkage analysis in affected family pedigrees and exome sequencing to identify mutations in KCND3, which encodes voltage-gated potassium channel $\mathrm{K}_{\mathrm{v}} 4.3$ subunit-a protein that is important in membrane repolarization.

\section{4 ...mutations in KCND3 ... might underlie ... SCA19 and SCA22 77}

"We screened through numerous candidate genes without success," explains Bing-wen Soong, who led one of the teams, "until we performed exome sequencing to pinpoint a defect in ... KCND3, which is implicated in cardiac arrhythmia but not known to cause neurological diseases."

SCAs are a hetereogenous group of neurodegenerative disorders characterized by extrapyramidal and peripheral nervous system involvement and autosomal dominant inheritance. The disease loci for SCA22 and SCA19 were previously mapped to chromosome 1p21-q21 by linkage analysis in large Chinese and Dutch kindreds, respectively. SCA19 and SCA22 disease loci overlap, and the disease-causing mutations were previously thought to be allelic.

Rare disorders such as the SCAs often occur in small pedigrees, in which traditional genetic analysis cannot identify the genes involved. Margit Burmeister, lead researcher in one of the groups that identified KCND3 mutations in SCA22, highlights how things have changed: "The new methodology of combining traditional genetic linkage analysis ... with whole-exome sequencing ... allows narrowing down and ultimately identification of the mutation."

The researchers led by Dineke Verbeek and Soong independently identified mutations in the KCND3 gene in the original SCA19 and SCA22 families. Burmeister's group and Giovanni Stevanin's group also identified new mutations in two other families. Three further mutations in KCND3 were identified in Japanese SCA22 kindreds by Shoji Tsuji's group. Notably, none of the mutations identified in SCA19 and SCA22 families was found in healthy controls.
Wild-type $\mathrm{K}_{\mathrm{v}} 4.3$ protein is associated with the plasma membrane, whereas the mutated protein found in patients is retained in the endoplasmic reticulum. Electrophysiological studies showed that mutations in KCND3 impair both channel function and neuronal excitability, suggesting these changes might underlie the slowly progressive cerebellar neurodegeneration observed in SCA19 and SCA22.

Burmeister's group are constructing a digital network of ataxia-related proteins, with the aim of classifying SCAs according to the pathways affected. "About four or five different pathways lead to ataxia," notes Burmeister. "Finding the new genes may open up new pathways," This approach, she suggests, might enable diagnosis of genetic defects in individuals without a family history of SCA — still the majority of patients with ataxia.

Ellen Bible

Original articles Lee, Y.-C. et al. Mutations in KCND3 cause spinocerebellar ataxia type 22. Ann. Neurol. doi:10.1002/ana.23701 | Duarri, A. et al. Mutations in potassium channel KCND3 cause spinocerebellar ataxia type 19. Ann. Neurol. doi:10.1002/ana.23700 